\title{
ENVIRONMENTS OF DYNAMIC LEARNING UNDER 3D INTERACTIVE VISION: RECONSTRUCTION OF THE ORBIT
}

\author{
Beatriz Framiñán Aparicio ${ }^{1}$, Juan Antonio Juanes Méndez ${ }^{2}$, \\ \& Andrés Framiñán de Miguel ${ }^{1}$ \\ ${ }^{I}$ Complejo Asistencial Universitario de Salamanca (Spain) \\ ${ }^{2}$ Universidad de Salamanca (Spain)
}

\begin{abstract}
Introduction: technology, applied to training in health sciences, has undergone great changes over time. These changes always occurred parallel to the technological evolution of society, which is why technological advances have modified the ways of teaching and learning of university students in experimental sciences and health. Between the different subjects in medicine, one of the most important is anatomy. This will form a base which will follow the students throughout their career.

Previous findings: traditionally, anatomical dissection has been the basis of anatomical studies. Although it is a global and accepted method currently used in almost all medical universities, it has its limitations. An important disadvantage is that sometimes the structures in the human body are too small, in addition to the low availability of the object of study.

Later, using radiological sections to analyse and enlarge different structures started. The main problem with this technique is that, even though you can obtain larger images, these are two-dimensional images. In recent years important technological advances have been developed in the post-processing of radiological medical imaging.

Objectives: the aim of our study is to demonstrate the advantages of 3D reconstruction of the orbit in comparison to other ways of teaching and studying. These innovative techniques allow the representation of three-dimensional anatomical images using image diagnostic devices coupled with software capable of reconstructing any radiological section: axial, coronal or sagittal. In particular the orbit can obtain benefit from this system given its morphology: pyramidal shape, walls formed by 7 different cranial bones, small volume, large number of structures inside.

Methods: applied research; comparison, observation and analysis. Devices used: Toshiba Aquilion 64 TC (a whole body 64-slice CT scanner) using Vitrea software.

Results: the three-dimensional vision has become a crucial tool for the learning and understanding of many complex anatomical structures such as the orbit, enabling the introduction of novel techniques for the teaching and learning of anatomical models.

Conclusions: our research demonstrates the advantages of the 3D reconstruction of the orbit; visualization of each structure with its anatomical relationships, image enlargement, precise description, accessibility, morphological comprehension and improvement of spatial perception.
\end{abstract}

Keywords: Teaching, technology, 3D reconstruction, anatomy, orbit.

\section{Introduction}

In recent years there has been a deep technological development. This transformation translates into all areas of life: social, educational, professional ... That is why consequently it is also about learning. The relationship between the current context of society and technology has evolved rapidly, specially involving medicine and health systems. In university education, we begin to have different experiences and more chiefly in the teaching of medicine. The scope of activity, information and knowledge are particularly relevant in the health sciences, mostly in subjects such as human anatomy (a field shared by several specialities, not only medicine).

Nowadays students have new ways of exploration and representation of information. Mobile devices and tablets represent a real revolution specially among youth. That is why today these new lines of accessing knowledge maintain an outstanding position. There are new ways to access knowledge through 
different resources such as platforms or applications. These technological means are considered as complementary tools in academic learning and are an incentive for students.

Currently the transmission of knowledge is changing and the need for adaptation by teachers and students arises. New techniques of education represent a challenge for both teachers and students, but they also constitute a way of enriching knowledge and contributing quality to education. Society evolves more and more towards self-learning, leaving aside the master class and the classical methods of study, producing a displacement of them but not a replacement.

\section{Previous findings}

Universities and specifically anatomy subjects are increasingly making an inclusion of this emerging technology. Traditionally anatomical atlas, different books or anatomical plates have been used for study. Anatomical dissection has also been used for decades. Although its replacement is not intended, there are some disadvantages: limited accessibility, group study, life size structures... which can make it difficult to understand certain small anatomical areas where many structures such as the orbit converge.

Subsequently, with the technological development they began to introduce several advances such as radiodiagnostic images. These devices allow greater accessibility to the object of study and enlargement of the images, which facilitates the study. Its main problem continues being that they are two-dimensional images, with the limitations that this implies.

There are new learning styles that can be linked together without replacing the traditional ways of training in the area of human anatomy. 3D reconstruction is one of these new methods. This technique is possible thanks to the advances occurred in recent years in the post-processing of radiological images. The three-dimensional reconstruction for the creation of anatomical models is a great diversity of layers of digital information, more interactive, which through its use we can alter or enrich the information. All this provides greater efficiency and improves the quality of the teaching and learning process. They facilitate both learning and training in the medical field.

\section{Objectives}

The aim of our study is to demonstrate the advantages offered by three-dimensional reconstructions in the study of human anatomy and in particular the orbit. We believe that the orbit is a body area that can specially benefit from this technique given its small size and the number of anatomical structures it houses inside. It is a $30 \mathrm{~cm} 2$ volume bone cavity that contains and protects delicate structures that comprises the visual system of the central nervous system. It is delimited by 4 walls and 7 bones: frontal bone, sphenoid bone, zigomatic bone, lacrimal bone, etmoidal bone, maxillary bone and palatine bone.

Inside we find its main organ: the eyeball. In addition, there is a great variety of soft tissues: orbital fat, the 7 extraocular muscles (medial rectus, lateral rectus, superior rectus, inferior rectus, superior oblique, inferior oblique and levator muscle of the upper eyelid) and the intraorbital part of the optic nerve.

We also found blood vessels as the central artery of the retina, and nerve structures both sensitive (V and VII pair) and motor (III, IV, VI pairs) and other structures such as the lacrimal system.

\section{Methods}

For the elaboration of three-dimensional anatomical models, images obtained by different diagnostic imaging devices are used: computerized tomography (CT), magnetic resonance (MR) or even ultrasound (US). We have focused mostly on the first one, CT, specifically the model Toshiba Aquilon 64 (a whole body 64-slice CT scanner). This scanner has been built on Toshiba's Quantam Detector, a volumetric 64-slice CT scanner with 64 detector channels, 3D cone beam algorithms and volume reconstruction. CT emits multiple simultaneous X-rays from different angles. The tissues in a tomographic plane are assigned a density value proportional to their coefficient of absorption of xrays. The beams are detected after they have passed through the body creating a cross-sectional image, which will be weaker if they have passed through dense tissue. Either two or three dimensional images are digitally constructed from these density measurements. Orbital CT scans are usually obtained in $2 \mathrm{~mm}$ sections and for greater detail fine cuts at $1 \mathrm{~mm}$ intervals may be requested. Orbital images can be obtained in the axial plane, parallel to the course of the optic nerve; in the coronal plane, showing the eye, optic nerve, and extraocular muscles in cross section; or in the sagittal plane, parallel to the nasal septum.

The images used come from the radiodiagnosis service of the Complejo Asistencial Universitario de Salamanca and the Affidea center. 
Subsequently, for the processing of these images, different programs are used: OsiriX Lite, 3D slicer, vitrea software, amira; in our study for the production of anatomical reconstructions vitrea software was used as a tool. Vitrea software is a multi-modality advanced visualization system providing comprehensive applications in a variety of information techonology environments. This CT uses its software to reconstruct (reformat) any section in any direction (axial, coronal, or sagittal). By providing access to advanced clinical tools, Vitrea software enables physicians and students to have meaningful interactions wherever they are.

We value the opinion of students of health sciences degrees where the subject of Human Anatomy is taught through a satisfaction survey to evaluate their opinion. Thus, our objective is to analyze whether the practical study of anatomy with these recently incorporated technological resources is a motivating element in learning as well as their opinion on the effectiveness of these methods. The evaluation of our technological developments of teaching innovation will be considered from a double perspective: a formative and a summative evaluation. The criteria that we apply for the summative evaluation or results is both the quality of the technological environment from a technical, pedagogical and functional dimension (level of effectiveness for the achievement of the objectives set, the relevance of the learning, relation to the cost of tools) as the quality of the learning achieved.

From a formative evaluation approach, that is, with the aim of improving those aspects of the technological background of immersive environments that are detected weaker, we apply some criteria and indicators to be able to contrast and take the appropriate decisions.

The indicators and their objective measure would be satisfaction surveys on the quality of the environment, detecting strengths, weaknesses and suggestions for improvement, by the teachers involved in the innovative experience and by the students.

\section{Results}

The three-dimensional vision has become a crucial tool for the learning and understanding of many complex anatomical structures such as the orbit, enabling the introduction of novel techniques for the teaching and learning of anatomical models.

With anatomical reconstructions, all the spatial relationships of the orbit are better defined given its small and pyramidal size. Emphasizing on the small dimensions: entrance height $35 \mathrm{~mm}$, entrance width $40-45 \mathrm{~mm}$, medial wall length $40-45 \mathrm{~mm}$ and $40 \mathrm{~mm}$ depth, 3D reconstruction facilitates its morphological comprehension. Its limits are defined by its 4 walls:

- The roof of the orbit is formed by the orbital plate of the frontal bone and the lesser wing of the sphenoid bone. It is located adjacent to the frontal sinus and has some important landmarks such as the supraorbital notch or the lacrimal gland fossa, which can be perfectly distinguished with this method.

- The medial wall is composed of the frontal process of the maxillary bone, the ethmoid bone, the lacrimal bone and the lesses wing of the sphenoid bone. It is also located adjacent to two sinuses: ethmoid and sphenoid. And some of the most important landmarks that are perfectly defined are the frontoethmoidal suture and the entry of the anterior and posterior ethmoidal arteries.

- The lateral wall is composed of the zigomatic and sphenoid bone. The superior orbit fissure (an important area from an anatomic point of view) can be viewed with the three-dimensional reconstructions and all the structures that passed through it.

- Finally, the floor of the orbit is composed of the palatine, maxillary and zigomatic bone. Here we can perfectly see some important and tiny structures such us the infraorbital groove and the infraorbital canal.

We try to create a tool to adapt the teaching of anatomy of the orbit to current educational trends. In this way we intend to complement the practical study of anatomy with a currently didactic support element: 3D anatomical reconstruction.

\section{Conclusions}

- The creation of three-dimensional anatomical models facilitates the study of the structures of the orbit for both students and medical practitioners thanks to the development of a practical tool that allows an approach to the study of the orbit dynamically.

- Nowadays it is important the integration of new technologies in the didactic field including medical training. 
- It is clear that technological advances define the basis of modern education and offer us the possibility of using in the teaching of experimental sciences and health new resources that offer a more versatile strategy for the representation of knowledge, without losing the formation of the classical prespective.

- It is necessary to keep the teaching methods constantly updated.

- Some of the advantages of 3D reconstruction of the orbit are: visualization of each structure with its anatomical relationships, image enlargement, precise description, accessibility, morphological comprehension and improvement of spatial perception.

\section{References}

Cabero Almenara J., Barroso Osuna J., Obrador M. (2017). Realidad aumentada aplicada a la enseñanza de la medicina. Educación Médica, Elsevier, Volume 18.

Cantor L.B., Rapuano Ch.J., Cioffi G.A. (2016-2017.) Orbit, eyelids and lacrimal systems. San Francisco, CA: AAO, BSCS.

Fitzhugh A., Naveed H., Davagnanam I., Messiha A. (2016). Proposed three-dimensional model of the orbit and relevance to orbital fracture repair. Surgery Radiologic Anatomy, Volume 38, Pages 557-561.

Guze Ph.A. (2015). Using technology to meet the challenges of medical education. American clinical and climatological association, Volume 126.

Juanes Méndez J.A. (2016). Current status of new technologies in the teaching of experimental sciences and medicine in particular. Educación Médica, Elsevier, Volume 16.

Lizaraso Caparó L., Paredes Pérez N. (2015). Use of Technology in Medical Training. Horizonte Médico, Volume 15.

Mohammad Mosa A. S., Illhoi Yoo I., Sheets L. (2012). A Systematic Review of Healthcare Applications for Smartphones. BMC Medical Informatics and Decision Making, Volume 12: 67.

Pryor W., Zacharia T. (2018). Imaging of the human orbit. Operative Techniques in Otolaryngology, Volume 29.

Valeri G., Mazza F.A., Maggi S., Aramini D., La Riccia L., Mazzoni G., Giovagnoni A. (2015). Open source software in a practical approach for post processing of radiologic images. Radiology Medicine, Volume 120.

Wilkinson M.J. (2018). Anatomy of the human orbit. Operative Techniques in Otolaryngology, Elsevier, Volume 29, Pages 186-192. 